\title{
Circuit Implementation of Coronary Artery Chaos Phenomenon and Optimal PID Synchronization Controller Design
}

\author{
Cheng-Yu Yeh, Jen Shiu, and Her-Terng Yau \\ Department of Electrical Engineering, National Chin-Yi University of Technology, Taichung 411, Taiwan \\ Correspondence should be addressed to Her-Terng Yau, pan1012@ms52.hinet.net
}

Received 4 July 2012; Accepted 24 August 2012

Academic Editor: Jui-Sheng Lin

Copyright (C) 2012 Cheng-Yu Yeh et al. This is an open access article distributed under the Creative Commons Attribution License, which permits unrestricted use, distribution, and reproduction in any medium, provided the original work is properly cited.

This study aimed at the implementation and synchronization control of cardiac circuit. First, the MATLAB-Simulink was used to simulate the dynamic behavior of cardiac chaotic circuit, and simple electronic modules were used to implement the cardiac system. Then the Particle Swarm Optimization (PSO) was used to seek for the proportional, integral, and derivative gains of optimal PID controller, and the PID controller which could synchronize the slave cardiac circuit and the master cardiac circuit was obtained, in order to synchronize the master/slave chaotic cardiac circuits. This method can be provided for cardiac doctors to diagnose and medicate cardiac abnormality.

\section{Introduction}

The mortality from coronary artery disease is always high in Taiwan or abroad. There are many causes for coronary artery disease, such as age, gender, family heredity, diabetes history, hypertension, and hyperlipemia [1, 2]. Many developed countries have exerted considerable efforts on studies of prevention and treatment of coronary artery diseases, especially with the present developed biomedical engineering. Many studies and methods have obvious treatment effect. It is difficult to control the nonlinear behavior of coronary artery, because it involves hemodynamics, constriction of wall, and the kinestate of muscles. From the angle of medicine, the cardiac chaotic behaviors must be synchronized, so as to improve the pathological change in cardiovascular system.

The theory and application of synchronization of chaotic systems have become popular research subjects in recent years, because many problems in daily life are usually concerned with synchronization of chaotic systems, for example, the traffic flow control on 
freeways during peak hours, the traffic stream on the freeway is the primary system, and the upstream and downstream on each interchange are the secondary system. If the primary system and the secondary system are synchronized, namely, the traffic flow and rate are controlled, there will be no traffic jam. In the sea, how to connect large ships to small ships in the fluctuant waves smoothly for oiling and transferring goods can be solved by such a concept of "synchronization." Even in the field of medicine, it can be used to adjust the heart rhythm regulator, so as to stabilize the heart beat frequency within the safety limit with the physical state to avoid heart attack. However, it is difficult to synchronize two nonlinear systems, and the selection of control mode is one of the factors. The two synchronous chaotic systems are called master system and slave system, respectively. In the generation of chaotic system, the slight difference in the initial values may result in voltage fluctuation and tremendous changes, occurring in a short or long duration, so the control mode of chaotic synchronization is the key point of this paper. Many recent studies have proposed different control modes based on chaotic synchronization. Lin et al. proposed synchronization control of time delay of complicated system dynamics based on adaptive robust observerbased approach [3]. Dismassi and Loría proposed synchronization control of the security of communication signals based on adaptive control [4]. However, all controllers require control parameters, so the search of parameters becomes another problem that must be overcome. In recent years, with the development of artificial intelligence, many studies have searched for the optimal parametric solution based on artificial intelligence. Sun et al. proposed a Neural Network based on PSO (particle swarm optimization) to predict and control the chaotic dynamic system for adaptive optimal control. The research result showed when the chaotic dynamic system is defined by the self-regulating function, the system could be stabilized [5]. Kao et al. proposed an adaptive dynamic neural network control to synchronize the chaotic gyros [6]. According to the previous research methods, using artificial intelligence to search for parameters and predict parameters can realize effective control and prediction. Therefore, this study uses PSO to seek for $k_{p}, k_{i}, k_{d}$ parameter solution of PID controller.

The particle swarm optimization (PSO) was proposed by Fernandez-Martinez and Garcia-Gonzalo Eberhart (2011), an optimization algorithm derived from "social psychology" and "Evolutional Computation" [7]. The social psychology discusses the interactive relationship between individual behavior and the society in the social situation, such as social adaptation, communication ability, and so forth. It is an interpersonal interactionoriented psychology. The behavior of individual interacts with others and then interacts with the whole society. The animals with social behavior in the nature, such as bird flocks, fish schools, and insects, transfer messages to each other to attain the goals of individual and colonies, such as food finding and gathering. The PSO was originally intended to simulate a simplified social behavior, such as the flight lineup of a bird flock or the gathering of a fish school. A swarm of particles are scattered in the search space, and each particle simulates a real organism, providing a mechanism for mutual communication and information exchange among particles in the swarm in the concept of information sharing in the biosociety. This is similar to social behavior of human and the behavior of other social organisms, so that these particles can search for approximate solution more effectively. In recent years, the PSO algorithm has been successfully applied to many research and application domains, and many research results have shown that the PSO algorithm can find the required optimal solution faster, because the PSO algorithm only needs parameter adjustment before it is widely used in multiple domains. The PSO is also widely used in biomedical engineering. Hedeshi and Abadeh proposed the medical image registration algorithm based on PSO as the method for coronary artery disease diagnosis, and the accuracy rate was as high as $94.37 \%$ [8]. 
Hedeshi and Abadeh proposed the coronary artery disease diagnosis expert system based on PSO again, with medical diagnostic data, and compared it with 14 expert systems including neural network and fuzzy expert system. The results showed that the diagnosis rate of the PSO-based expert diagnosis system is as high as $92.59 \%$, and then the neural network expert system is $89.01 \%$ [9].

Many experts and scholars have proposed various control models for the synchronization control of cardiac chaos. Brandt and Chen proposed the self-tuning feedback control for controlling arrhythmia, and the experiment showed that the lagging feedback of arrhythmia time was improved promptly [10]. Gong et al. proposed the synchronization control based on backstepping for controlling myocardial infarction and vasospasm and proved that the backstepping synchronization control could synchronize the spasmodic and normal cardiovascular systems for treatment [11]. Li and Yuan proposed the nonlinear and chaotic characteristics based on heart sounds to control the time series of splitting of heart sounds mitral insufficiency and aortic constriction abnormity. The control theory used fractal dimensions and Lyapunov exponents. The results showed that the healthy cardiac chaos has more obvious chaotic motion than the abnormal cardiac chaos [12]. Li proposed the selfadaptive robust control tracking chaotic coronary artery system, and the simulation results showed that the chaotic coronary artery system enters the normal orbit [13].

This study used the simple PID controller first as the control mode for suppressing pathological heartbeat, so that the cardiac chaotic behavior would reach master/slave synchronization. The proportional, derivative, and integral $k_{p}, k_{i}, k_{d}$ gains of PID controller must be very precise, so as to synchronize the pathological heart and the healthy heart perfectly and to reduce the incidence of myocardial infarction. This study used PSO to seek for the optimal solution for proportional, differential, and integral gains. According to the simulation results of this experiment, using the controller parameters obtained using PSO in the PID controller can synchronize cardiac chaotic system effectively, meaning that the control signal $u(t)$ can suppress pathological heart effectively from the angle of medicine, $u(t)$ is the medicine for treating cardiac pathological changes, such as nitrates and calcium ion blocking agent. The pathological heart can be controlled and the incidence can be reduced easily as long as $u(t)$ is controlled appropriately.

\section{Cardiac Chaos Phenomenon}

In cardiodynamics, the cardiac motion of human has a certain chaos, and the disappearance of pathological chaos phenomenon is the symptom of some heart diseases on the contrary. In this case, this chaos phenomenon should be maintained or enhanced to maintain the normal function of heart. About 400,000 people died of heart disease in the USA yearly and most of them died of irregular physiological reaction caused by ventricular fibrosis. A single throb in this reaction can change the heartthrob from regular contraction into spasmic and fatal spasm. This chaotic irregular heartbeat phenomenon is cardiac chaos.

A mathematical model can be obtained from cardiodynamics to simulate cardiac chaos phenomenon, and the equation is expressed as [13]

$$
\begin{gathered}
\dot{x}=-b x-c y, \\
\dot{y}=-(\lambda+b \lambda) x-(\lambda+c \lambda) y+\lambda x^{3}+E \cos (\omega t)+u .
\end{gathered}
$$




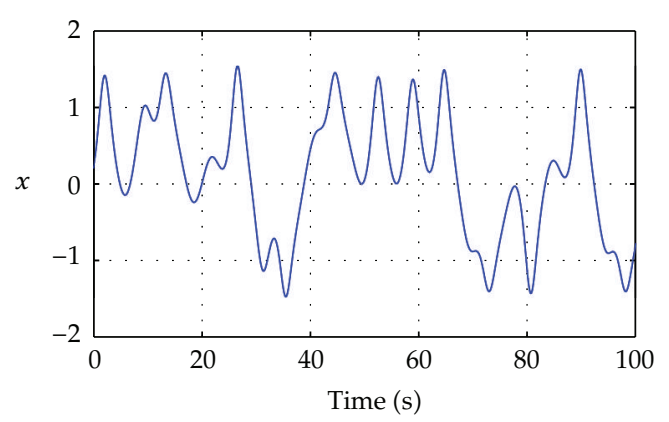

(a)

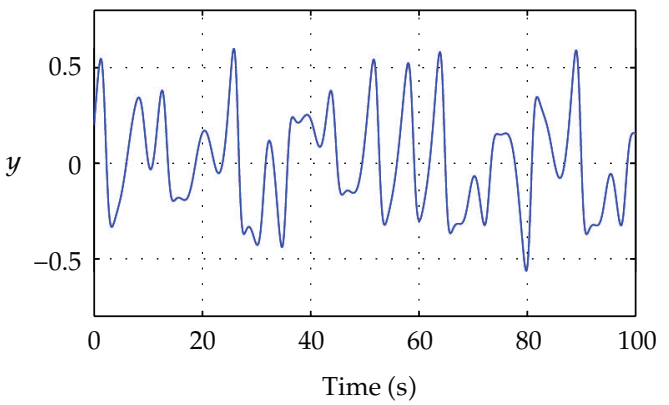

(b)

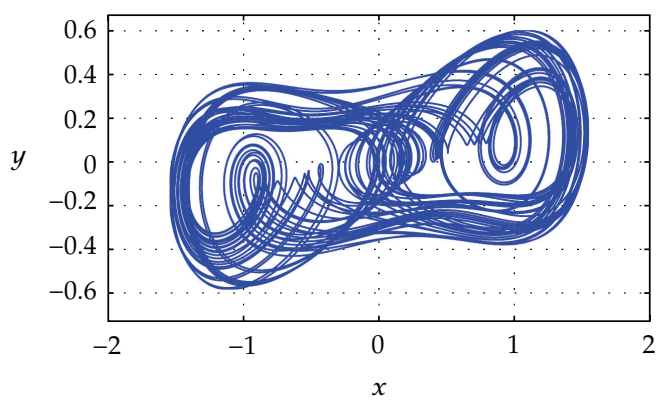

(c)

Figure 1: (a) Time simulation response diagram of $x$, (b) time simulation response diagram of $y$, and (c) simulation plan view of $x$ and $y$.

when parameters $b=0.15, c=-1.7, \lambda=-0.65, E=0.3, \omega=1$, and $u=0$ are substituted in (2.1) we obtain

$$
\begin{gathered}
\dot{x}=-0.15 x+1.7 y, \\
\dot{y}=0.7475 x-0.455 y+0.65 x^{3}+0.3 \cos (t) .
\end{gathered}
$$

The initial condition of (2.2) is set as 0 , and then the cardiac chaotic behavior can be simulated by MATLAB. Figure $1(\mathrm{a})$ is the time simulation response diagram of $x$, Figure 1(b) is the time simulation response diagram of $y$, and Figure 1(c) is the simulated plan view of $x$ and $y$.

Equation (2.2) is used in PSpice simulation software to draw the cardiac circuit diagram. Simple electronic modules are used to make the simulated heart model, and the oscillograph is used to observe the chaotic behaviors of $x$ and $y$. Figure 2(a) shows the PSpice cardiac chaotic circuit diagram, Figure 2(b) the time response diagram of simulated cardiac chaos, Figure 2(c), the time response diagram of simulated cardiac chaos $y$, and Figure 2(d), the plan view of $x$ and $y$ of simulated cardiac chaotic circuit.

\section{Cardiac Chaos Synchronization}

A master and a slave must be defined for cardiac chaos synchronization, and the chaotic behavior can be changed by changing the initial value of slave cardiac system. The chaotic 


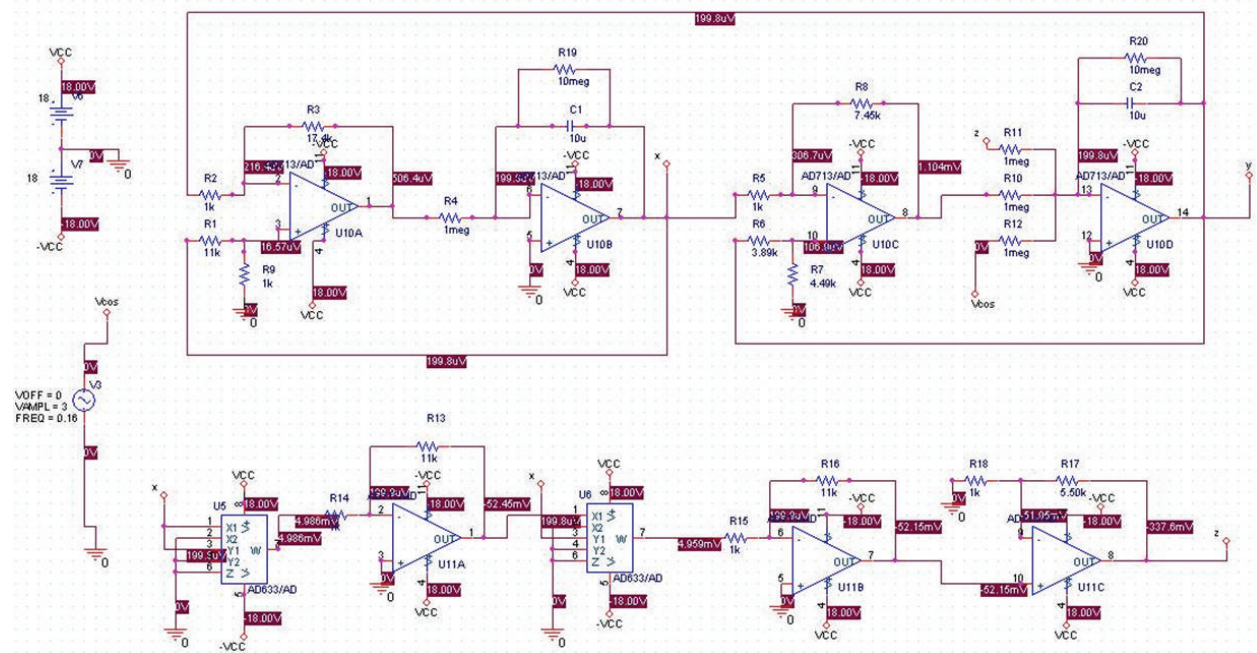

(a)

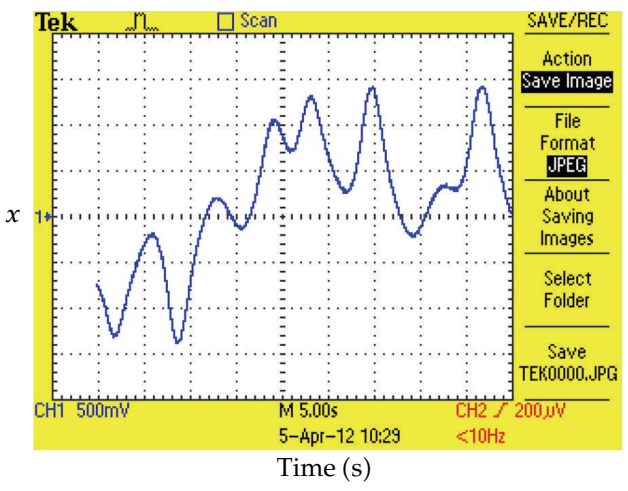

(b)

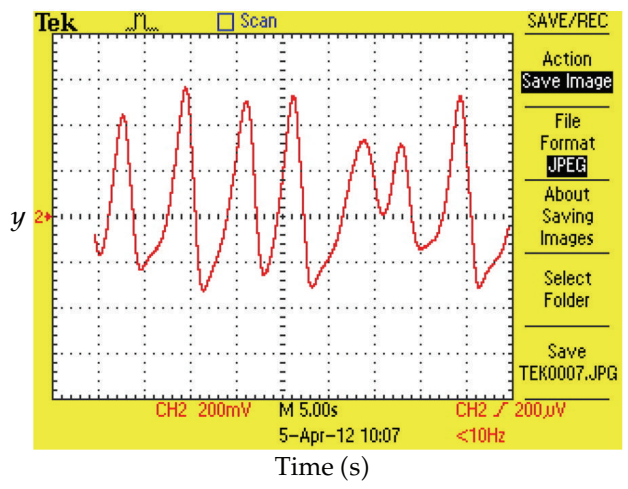

(c)

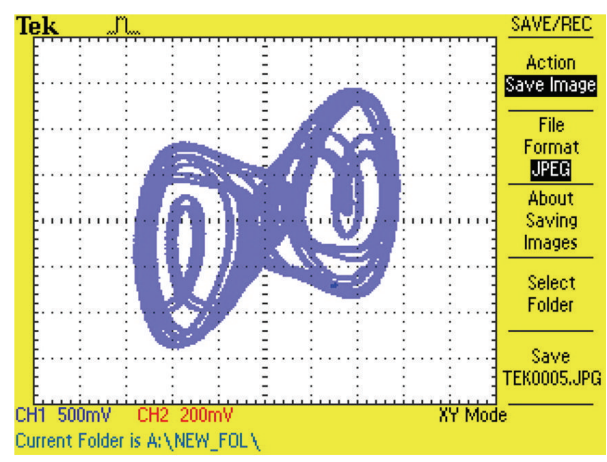

(d)

Figure 2: (a) PSpice cardiac chaotic circuit diagram, (b) oscillograph time response diagram of simulated cardiac chaos state $x,(\mathrm{c})$ oscillograph time response diagram of simulated cardiac chaos state $y$, and (d) oscillograph plan view of simulated cardiac chaotic circuit states $x$ and $y$. 
behavior of this slave cardiac chaotic behavior is like the pathological heart, and then the control signal of $u(t)$ is added to the slave cardiac system as the signal output of PID controller; the master and slave equations are (3.1) and (3.2).

Coronary artery master system:

$$
\begin{gathered}
\dot{x}_{M}=-0.15 x_{M}+1.7 y_{M^{\prime}} \\
\dot{y}_{M}=0.7475 x_{M}-0.455 y_{M}+0.65 x_{M}{ }^{3}+0.3 \cos (t) .
\end{gathered}
$$

Coronary artery slave system:

$$
\begin{gathered}
\dot{x}_{S}=-0.15 x_{M}+1.7 y_{s^{\prime}} \\
\dot{y}_{S}=0.7475 x_{s}-0.455 y_{S}+0.65 x_{s}{ }^{3}+0.3 \cos (t)+u(t),
\end{gathered}
$$

where $u(t)$ is the control signal, as shown in (3.3), for impelling the slave system to track the master system for synchronization. The controller $u(t)$ is defined as

$$
u(t)=f\left(y_{S}-y_{M}\right)
$$

The controller $u(t)$ is changed into the standard form of PID controller, then the equation is expressed as [14]

$$
u(t)=k_{p}\left[y_{S}(t)-y_{M}(t)\right]+k_{i} \int_{0}^{t}\left[y_{S}(t)-y_{M}(t)\right] d t+k_{d} \frac{d\left[y_{S}(t)-y_{M}(t)\right]}{d t} .
$$

In general cases, the adjustment of PID controller is how to select proper parameters $K_{p}, K_{i}, K_{d}$ to ensure the system with better control performance, and the performance standard (objective function) can be defined according to the required specifications. There are two performance indexes, Integrated Squared Error (ISE) and Integrated Absolute Error (IAE). Their mathematical definitions are shown below:

$$
\begin{aligned}
\mathrm{ISE} & =\int_{0}^{\infty} e^{2}(\tau) d \tau . \\
\mathrm{IAE} & =\int_{0}^{\infty}|e(\tau)| d \tau .
\end{aligned}
$$

This paper uses IAE as the objective function (OF), and the error state vector is $E(t)=$ $\left[x_{S}-x_{M}, y_{S}-y_{M}\right]$. Therefore, (3.6) is changed to the following equation:

$$
\mathrm{OF}=\mathrm{IAE}=\int_{0}^{\infty}|\|E(\tau)\|| d \tau .
$$

According to PSO algorithm, an ideal gain parameter adjustment method for PID controller is determined to minimize the objective function. 


\section{Introduction to Particle Swarm Optimization}

\subsection{Outline of Particle Swarm Optimization}

The PSO is a global optimal solution search algorithm based on the population concept. The whole process is like simulating real organisms, such as flight of bird flocks or gathering of fish schools, and it has common characteristics of Evolutional Computation concept, such as evaluation of adaptability. Compared with GA, PSO regards many search solutions as a simulated organism population and uses random number to initialize the whole population at the beginning. Whether the particles move towards individual optimal experience or population optimal experience is also similar to the concept of "crossover" operator of GA. However, the particle in PSO, that is, each possible potential solution, has velocity and "flies" in the whole search space; meanwhile, the particles and particle swarm have ability to memorize. These behaviors and abilities are completely different from GA.

\subsection{Principle of PSO}

The PSO algorithm is based on population. The individuals of the population are moved to good regions according to the adaptability to the environment. However, it does not use evolution operator for individuals, but regards each individual as a volumeless particle (point) in $D$-dimensional search space, flying at a certain velocity in the search space. This velocity is adjusted dynamically according to its flight experience and others' flight experience. The $i$ th particle is expressed as $X_{i}=\left(x_{i 1}, x_{i 2}, \ldots, x_{i D}\right)$. The best position it has passed through has the best adaptive value, expressed as $P_{i}=\left(p_{i 1}, p_{i 2}, \ldots, p_{i D}\right)$, also known as $p$ best. The index number of the best position passed through by all particles in the population is expressed as symbol $g$, that is, $P_{g}$, also known as gbest. The velocity of particle $i$ is expressed as $V_{i}=\left(v_{i 1}, v_{i 2}, \ldots, v_{i D}\right)$. The dimension $d(1 \leq d \leq D)$ of each generation is changed according to

$$
\begin{gathered}
v_{i d}=w \times v_{i d}+c_{1} \times \operatorname{Rand}() \times\left(p_{i d}-x_{i d}\right)+c_{2} \times \operatorname{Rand}() \times\left(p_{g d}-x_{i d}\right) . \\
x_{i d}=x_{i d}+v_{i d} .
\end{gathered}
$$

Equation (4.1) is velocity renewal function, while (4.2) is renewal position function, where $v_{i d}$ is the velocity of each particle in dimension $\mathrm{d}, i$ the number of particle, $d$ the dimension, $w$ the inertia weight, $c_{1}, c_{2}$ the learning constants, Rand( ) the random number between 0 and $1, P_{i d}$ the optimum position of each particle up to now, $P_{g d}$ the optimum position of all particles up to now, $x_{i d}$ and the present position of each particle.

The particles move according to (4.1) and (4.2), and then the corresponding adaptability is evaluated. The best position solutions met by each particle and whole particle swarm are recorded. When proper termination criteria are met, the recorded best position solution of particle swarm is the final optimal solution. Figure 3 shows the flow of PSO; the main procedure of PSO is described below [16].

(a) Determine swarm size and initialize position and velocity of each particle with random numbers.

(b) Evaluate the adaptability (OF) of potential solution for each, and update if the adaptability of this solution is better than the solution in individual memory. 


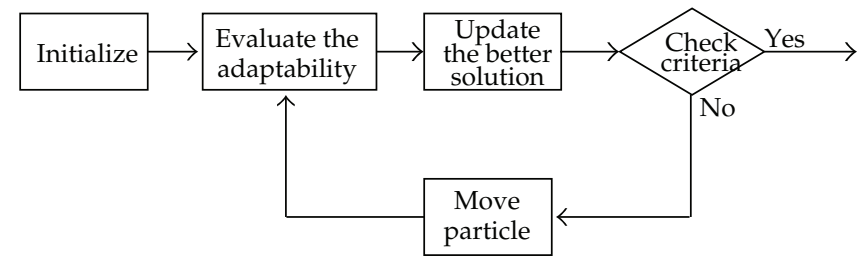

Figure 3: PSO flow.

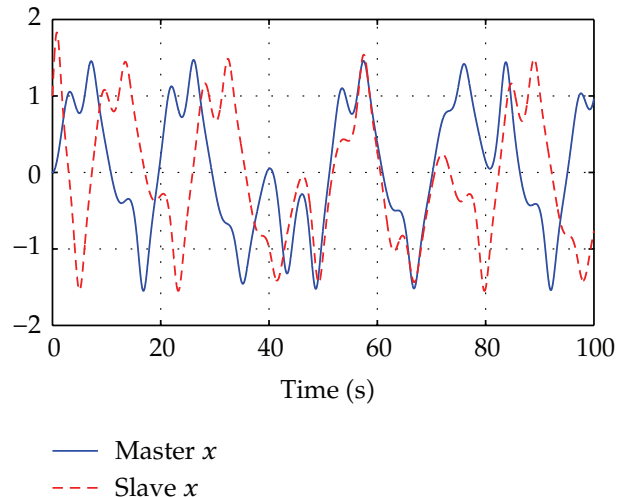

(a)

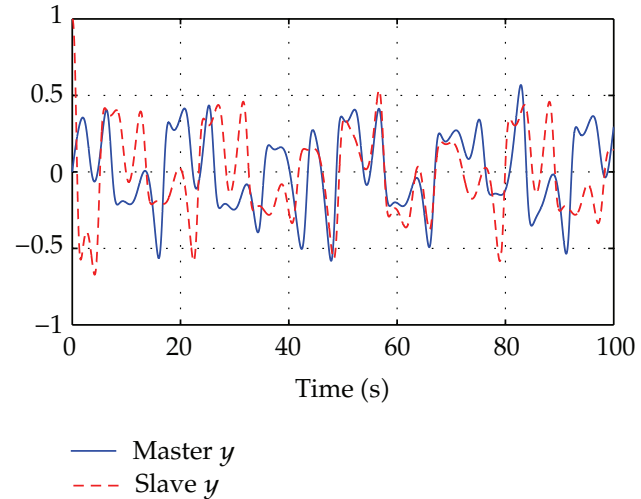

(b)

Figure 4: (a) Simulated time response diagram of $x_{m}$ and $x_{s}$ without PID control signal $u(t)$ and (b) simulated time response diagram of $y_{m}$ and $y_{s}$ without PID control signal $u(t)$.

(c) Find out the new best position solution in the whole swarm, and update if the adaptability of this solution is better than the solution in previous swarm memory.

(d) Finish PSO if the termination criteria are met.

(e) Equations (4.1) and (4.2) are used to update the velocity and position of each particle. Return to Step (2).

\section{Simulation Results}

The simulation software is MATLAB-Simulink and the mathematical expressions of (3.1) and (3.2) are used. The master system is healthy cardiac chaos phenomenon and the initial values of master system are set as $x_{M}(0)=0$ and $y_{M}(0)=0$. The slave system is pathological cardiac chaos, and the initial values of slave system are set as $x_{S}(0)=1$ and $y_{S}(0)=1$. The cardiac chaos master and slave systems without PID control signal $u(t)$ are used in PSO algorithm to search for the optimal $k_{p}, k_{i}, k_{d}$ parameter solution of PID controller. Figure 4(a) is the simulated time response diagram of $x_{M}$ and $x_{S}$ without PID control signal $(t)$, and $(\mathrm{b})$ is the simulated time response diagram of $y_{M}$ and $y_{S}$ without PID control signal $u(t)$. The PSO iterative search number is 50. Figure 5 is the convergence map of PSO searching for $k_{p}, k_{i}$, $k_{d}$ parameters. The IAE converges to a fixed value 0.461 after 10 iterations as calculated by PSO algorithm, as shown in Figure 6. According to Figure 5, after 10 iterations using PSO, $k_{p}$, $k_{i}, k_{d}$ parameter values are horizontal, meaning that PSO almost finds the optimal solution 


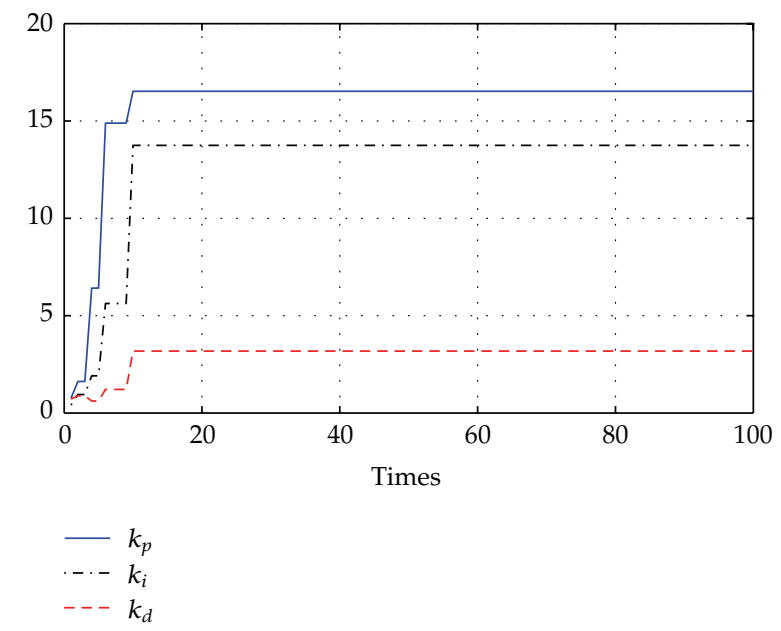

Figure 5: Convergence map of PSO searched $k_{p}, k_{i}, k_{d}$ parameters $\left(k_{p}=k_{i} 16.52,=13.75, k_{d}=3.18\right)$.

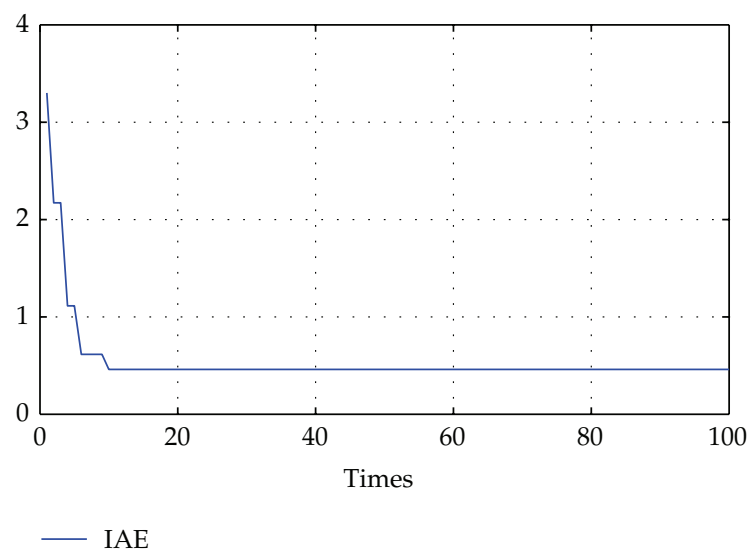

Figure 6: Converge curve of IAE.

of $k_{p}, k_{i}, k_{d}$ parameters after 10 iterations. This simulation used the parameters of the 50th iteration, $k_{p}=16.52, k_{i}=13.75, k_{d}=3.18$.

The $k_{p}, k_{i}, k_{d}$ parameters obtained by PSO are used in PID controller of cardiac chaotic system. The control signal $u(t)$ is added in at the 40th second. The numerical simulations of cardiac chaotic synchronization system with PID controller are shown in Figure 7. From Figure 7(c), the error states of master-slave cardiac chaotic synchronization system converge to 0 rapidly when the control signal $u(t)$ is added in at $40 \mathrm{sec}$. This PID controller can suppress pathological changes effectively for master-slave synchronization.

\section{Experimental Result}

The simulated cardiac chaotic circuit was used in the experiment, and the MATLABdSpace was used to design digital PID controller, the digital PID controller implemented synchronization control of the simulated cardiac chaotic circuit, and the PID controller 


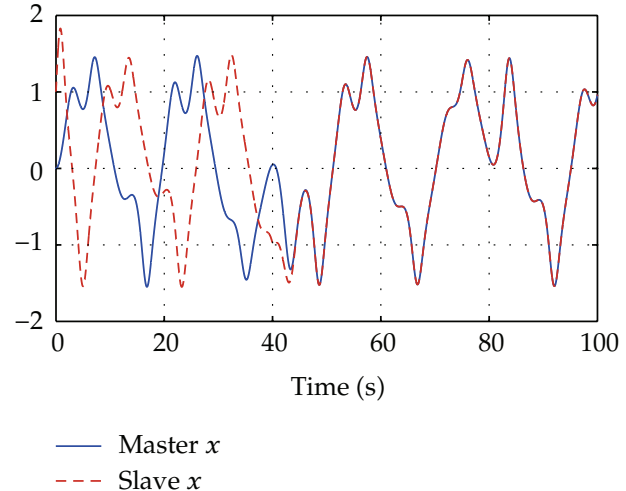

(a)

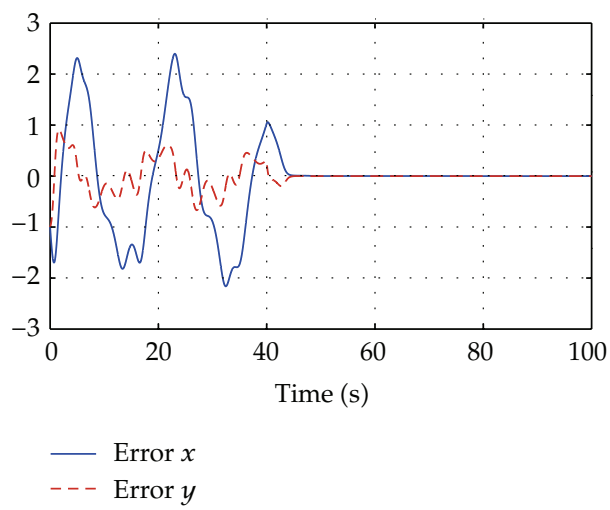

(c)

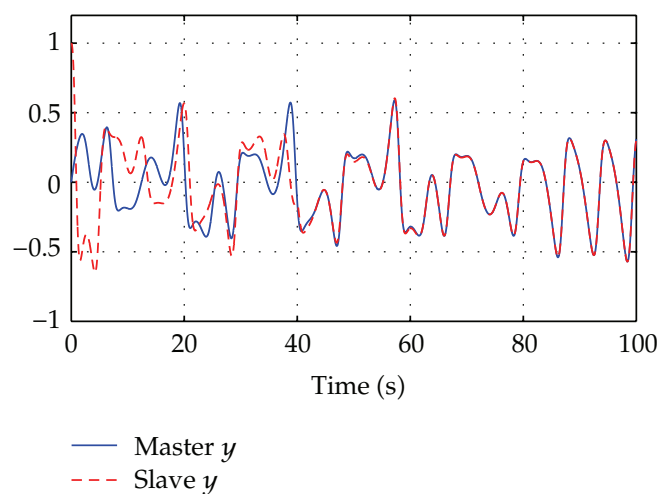

(b)

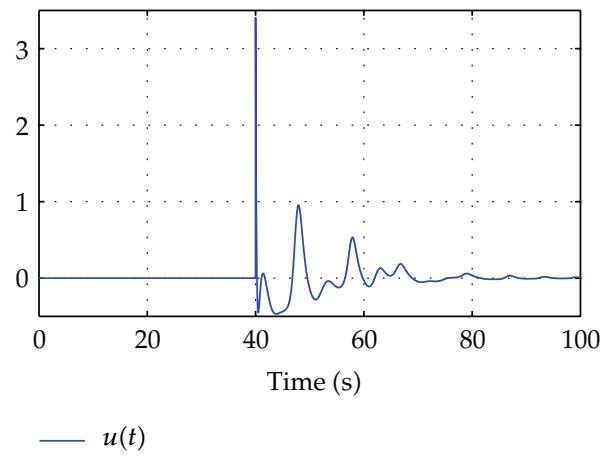

(d)

Figure 7: (a) Simulated time response diagram of $x_{m}$ and $x_{s}$ with PID control signal $u(t)$ at 40 sec, (b) simulated time response diagram of $y_{m}$ and $y_{s}$ with PID control signal $u(t)$ at $40 \mathrm{sec}$, (c) steady-state error convergence simulation curve of $x$ and $y$, and (d) simulated time response diagram of control signal $u(t)$.

parameters were simulated parameters, $k_{p}=16.52, k_{i}=13.75, k_{d}=3.18$. The PID control signal $u(t)$ was added in at $40 \mathrm{sec}$. The experimental result is shown in Figure 8(a): implemented time response diagram of $x_{M}$ and $x_{S}$ with PID control signal $u(t)$ at $40 \mathrm{sec}$, (b) implemented time response diagram of $y_{M}$ and $y_{S}$ with PID control signal $u(t)$ at 40 sec, (c) steady-state error convergence curve of $x$ and $y$, (d) implemented time response diagram of synchronous $x_{M}$ and $x_{S}$ of simulated heart, and (e) implemented time response diagram of synchronous $y_{M}$ and $y_{S}$ of simulated heart. According to the above experimental results, when the digital PID controller is added in the simulated cardiac chaotic circuit, the slave system becomes synchronous to the master system gradually. It proves that using the proportional, integral and differential gain parameters obtained by PSO in the PID controller can reach cardiac chaos synchronization control.

\section{Conclusion}

This study discussed the synchronization control of cardiac chaotic system based on a simple digital PID controller. The PSO was used to find the optimal solution of parameters $k_{p}, k_{i}, k_{d}$ 

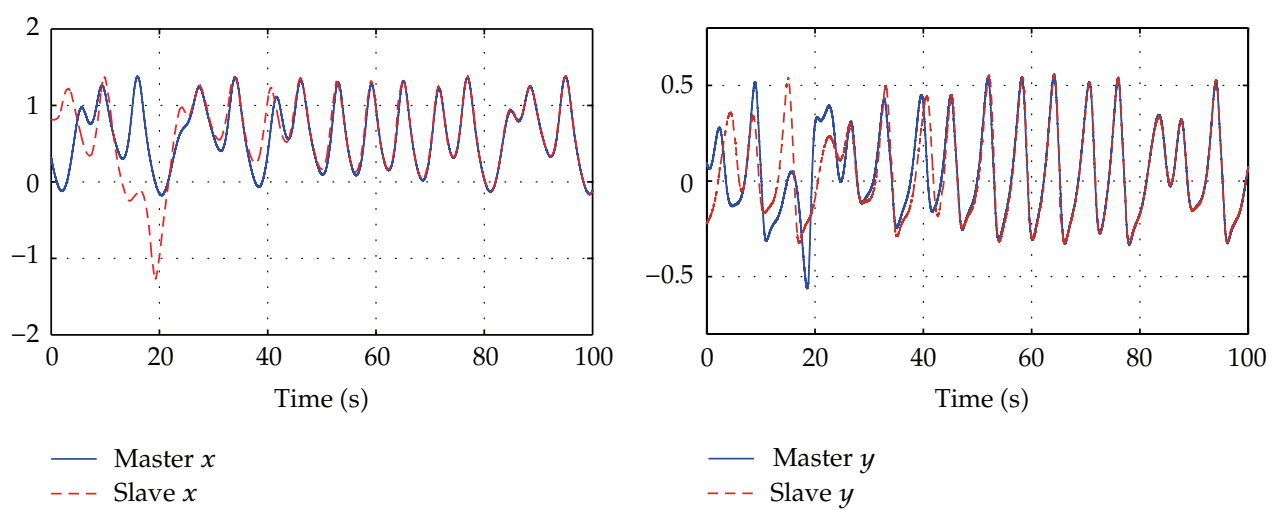

(a)

(b)

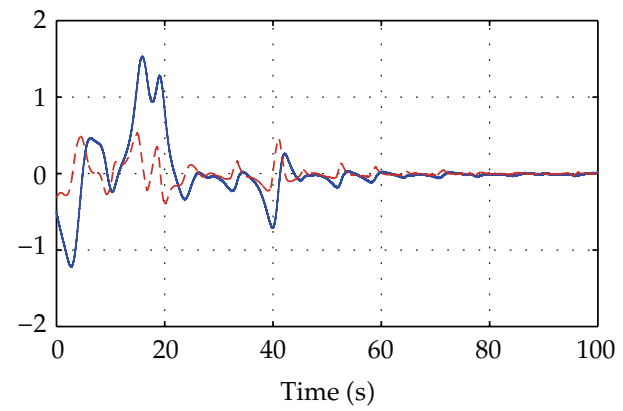

Error $x$
-- Error $y$

(c)

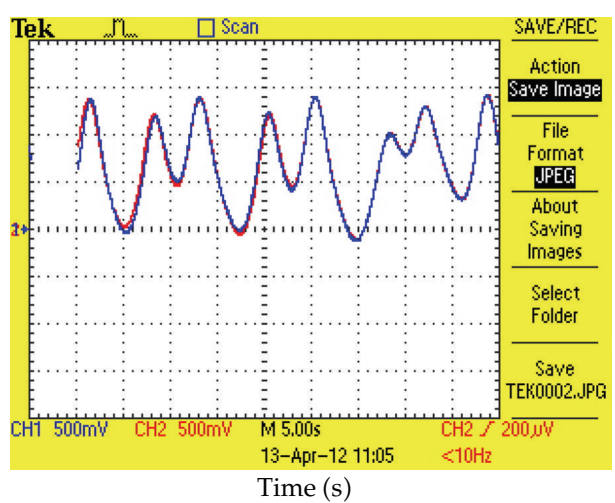

(d)

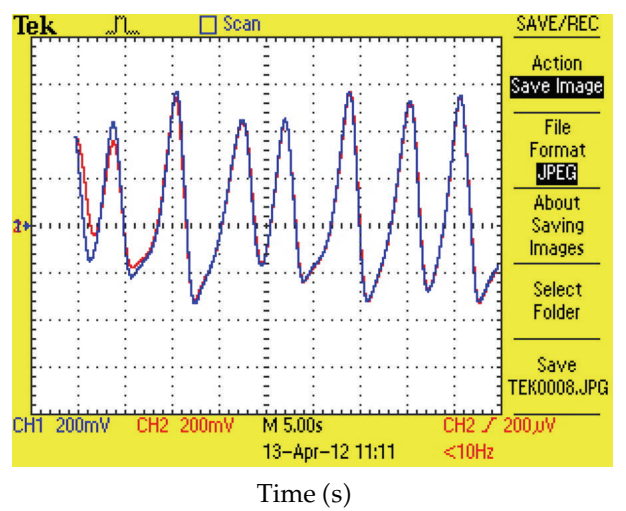

(e)

Figure 8: (a) Implemented time response diagram of $x_{m}$ and $x_{s}$ with PID control signal $u(t)$ at $40 \mathrm{sec}$, (b) implemented time response diagram of $y_{m}$ and $y_{s}$ with PID control signal $u(t)$ at $40 \mathrm{sec}$, (c) steady-state error convergence curve of $x$ and $y,(\mathrm{~d})$ oscillograph time response diagram of synchronous $x_{m}$ and $x_{s}$ of simulated heart, and (e) oscillograph time response diagram of synchronous $y_{m}$ and $y_{s}$ of simulated heart. 
of PID controller to optimize the PID controller. According to the simulation results, the PID controller parameters obtained by PSO can realize synchronization control of cardiac chaotic system effectively and accurately. It also proved the feasibility of optimal PID controller. The PSO simulated parameters were used in the experiment, and the digital PID controller was used for synchronization control of the simulated cardiac chaotic system. According to the experimental results, when the controller triggers the control signal $u(t)$ at $40 \mathrm{sec}$, the slave system can be synchronous to the master system promptly, thus proving the optimization of digital PID. In other words, a simple PID controller also can suppress pathological heart effectively. The pathological slave system with control signal $u(t)$ is like human body absorbing medicine, the blood vessel is expanded, more blood is supplied to cardiac muscle, and the angina pectoris symptom is relieved or eliminated for treatment. This experiment proved the optimization of digital PID controller, and it can be provided for doctors to suppress cardiovascular disease.

\section{Acknowledgment}

The financial support of this paper by the National Science Council of Taiwan, under Grant no. NSC 100-2628-E-167-002-MY3, is greatly appreciated.

\section{References}

[1] M. G. Tsipouras, T. P. Exarchos, D. I. Fotiadis et al., "Automated diagnosis of coronary artery disease based on data mining and fuzzy modeling," IEEE Transactions on Information Technology in Biomedicine, vol. 12, no. 4, pp. 447-458, 2008.

[2] H. Bagher-Ebadian, H. Soltanian-Zadeh, S. Setayeshi, and S. T. Smith, "Neural network and fuzzy clustering approach for automatic diagnosis of coronary artery disease in nuclear medicine," IEEE Transactions on Nuclear Science, vol. 51, no. 1, pp. 184-192, 2004.

[3] J.-S. Lin, T.-L. Liao, J.-J. Yan, and H.-T. Yau, "Synchronization of unidirectional coupled chaotic systems with unknown channel time-delay: adaptive robust observer-based approach," Chaos, Solitons and Fractals, vol. 26, no. 3, pp. 971-978, 2005.

[4] H. Dimassi and A. Loría, "Adaptive unknown-input observers-based synchronization of chaotic systems for telecommunication," IEEE Transactions on Circuits and Systems, vol. 58, no. 4, pp. 800-812, 2011.

[5] X. Sun, C. Yun, Y. Cui, and Z. An, "Controlling chaos by PSO-based neural network," in Proceedings of the International Conference on Intelligent Human-Machine Systems and Cybernetics (IHMSC '09), vol. 1, pp. 249-252, August 2009.

[6] C.-H. Kao, C.-F. Hsu, C.-H. Wang, and H.-S. Don, “Chaos synchronization using adaptive dynamic neural network controller with variable learning rates," Mathematical Problems in Engineering, vol. 2011, Article ID 701671, 20 pages, 2011.

[7] J. L. Fernandez-Martinez and E. Garcia-Gonzalo, "Stochastic stability analysis of the linear continuous and discrete pso models," IEEE Transactions on Evolutionary Computation, vol. 15, no. 3, Article ID Article number5675669, pp. 405-423, 2011.

[8] N. G. Hedeshi and M. S. Abadeh, “An ensemble PSO-based approach for diagnosis of coronary artery disease," in Proceedings of the International Symposium on Artificial Intelligence and Signal Processing (AISP'11), pp. 77-82, 2011.

[9] N. G. Hedeshi and M. S. Abadeh, "An expert system working upon an ensemble PSO-based approach for diagnosis of coronary artery disease," in Proceedings of the 18th Iranian Conference of Biomedical Engineering (ICBME '11), pp. 249-254, 2011.

[10] M. E. Brandt and G. Chen, "Feedback control of pathological rhythms in two models of cardiac activity," in Proceedings of the 1st International Conference on Control of Oscillations and Chaos (COC '9), pp. 219-223, August 1997. 
[11] C. Y. Gong, Y. M. Li, and X. H. Sun, "Control of synchronization for biomathematical model of muscular blood vessel," Journal of Applied Sciences, vol. 24, no. 6, pp. 604-607, 2006.

[12] B. B. Li and Z. F. Yuan, "Non-linear and chaos characteristics of heart sound time series," Proceedings of the Institution of Mechanical Engineers, Part H, vol. 222, no. 3, pp. 265-272, 2008.

[13] W. Li, "Tracking control of chaotic coronary artery system," International Journal of Systems Science, vol. 43, no. 1, pp. 21-30, 2012.

[14] A. Rubaai, M. J. Castro-Sitiriche, and A. R. Ofoli, “Design and implementation of parallel fuzzy PID controller for high-performance brushless motor drives: an integrated environment for rapid control prototyping," IEEE Transactions on Industry Applications, vol. 44, no. 4, pp. 1090-1098, 2008.

[15] B. Liu, L. Wang, and Y. H. Jin, "An effective PSO-based memetic algorithm for flow shop scheduling," IEEE Transactions on Systems, Man, and Cybernetics, Part B, vol. 37, no. 1, pp. 18-27, 2007.

[16] X. Chen and Y. Li, "A modified PSO structure resulting in high exploration ability with convergence guaranteed," IEEE Transactions on Systems, Man, and Cybernetics, Part B, vol. 37, no. 5, pp. 1271-1289, 2007. 


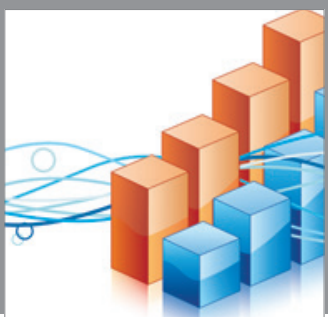

Advances in

Operations Research

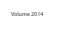

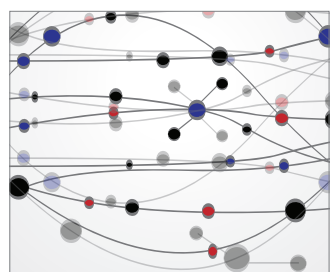

\section{The Scientific} World Journal
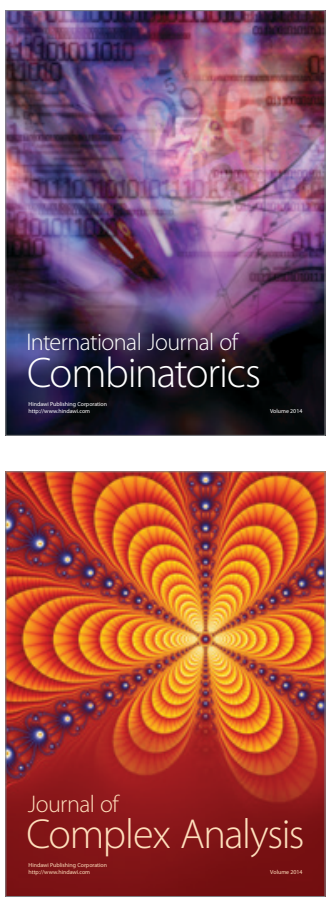

International Journal of

Mathematics and

Mathematical

Sciences
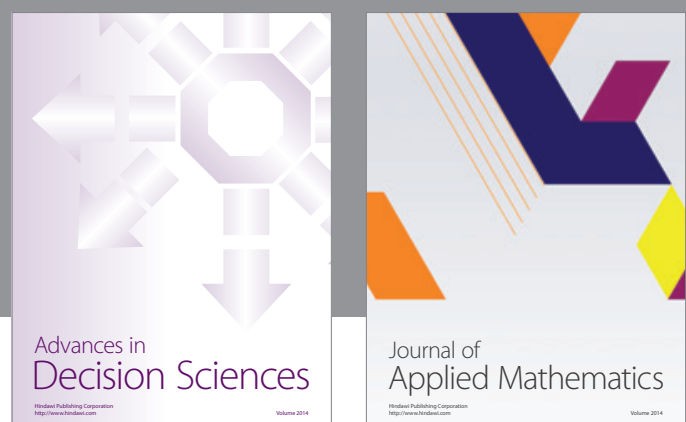

Journal of

Applied Mathematics
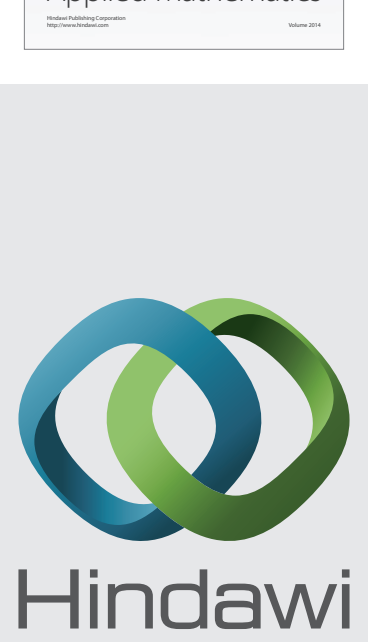

Submit your manuscripts at http://www.hindawi.com
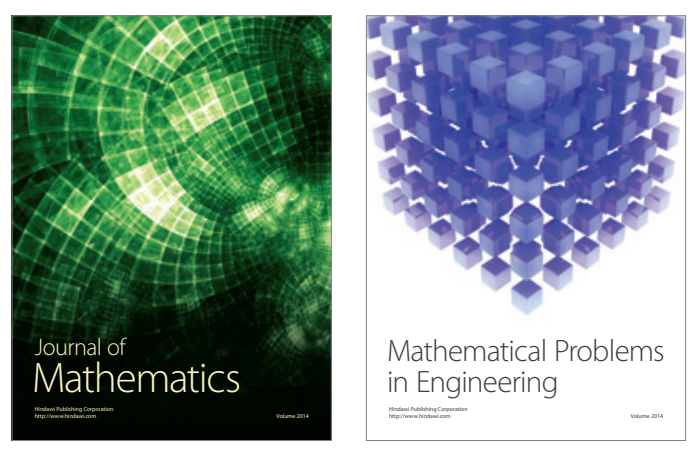

Mathematical Problems in Engineering
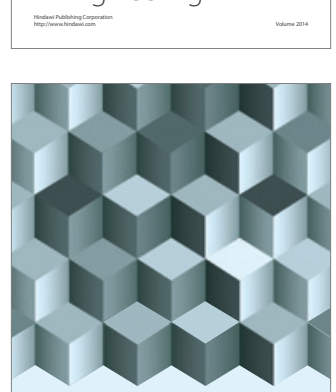

Journal of

Function Spaces
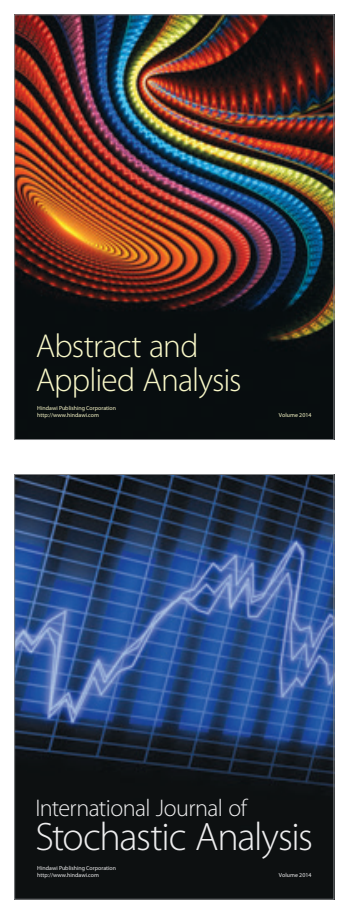

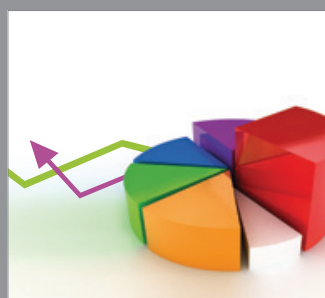

ournal of

Probability and Statistics

Promensencen
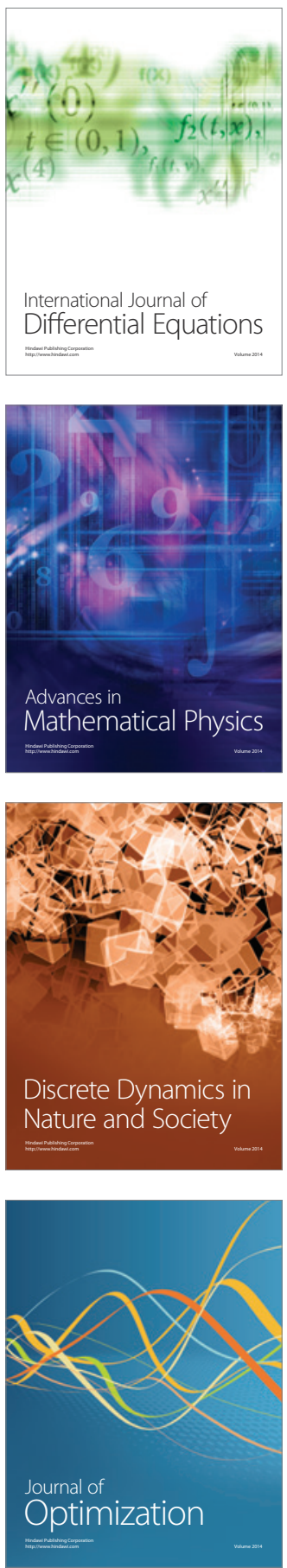